\title{
Noise of short-time integrators for readout of uncooled infrared bolometer arrays
}

\author{
D. Würfel, D. Weiler, B. J. Hosticka, and H. Vogt \\ Fraunhofer Institute for Microelectronic Circuits and Systems, Duisburg, Germany
}

\begin{abstract}
As state-of-the-art readout circuits short-time integrators in Far Infrared (FIR) uncooled bolometer arrays are commonly used. This paper compares the transfer functions of an ideal continuous-time integrator with that of a real integrator with focus an OTA parameters and noise analysis. Beside the noise sources at the non-inverting input of the OTA special care has to be taken to account for capacitances at the inverting input. The Noise Equivalent Temperature Difference (NETD) as the key parameter for bolometer applications for a real short-time integrator will be derived. As the result it will be shown, that the NETD is $1 / f$-noise limited.
\end{abstract}

\section{Introduction}

The need for uncooled infrared focal plane arrays (IRFPA) for imaging systems has increased since the beginning of the nineties. Examples for the application of IRFPAs are thermography, pedestrian detection for automotive, firefighting and infrared spectroscopy. According to Plank's law, each body with a temperature above absolute zero point emits electro-magnetic radiation. The wavelength and intensity depends on the temperature of the body. For example, a body at a temperature of $300 \mathrm{~K}$ has its maximum emittance at a wavelength of approx. $10 \mu \mathrm{m}$. FIR imager uses the IR-radiation of a warm body in the wavelength range between $8.14 \mu \mathrm{m}$. The FIR-sensitive element is the bolometer which changes its resistance by absorbing the radiation of the warm body. A pulsed bias with a constant voltage is applied to the bolometer and the resulting current is integrated for a fixed time. An offset current is subtracted before integration. Conventional readout circuits use a column-wise architecture.

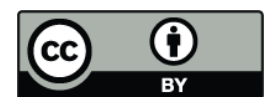

Correspondence to: D. Würfel (daniel.wuerfel@ims.fraunhofer.de)

\section{Bolometer}

The bolometer is fabricated by post-processing on CMOS wafers in Fraunhofer IMS Microsystem Lab. The realization of a microbolometer is shown in Fig. 1 as a cross section. A micromachined membrane consisting of amorphous silicon is suspended by two via stacks of metal from the CMOS substrate. The membrane forms with two other layers a good interferometric structure for radiation absorption. On top of the membrane an antireflection layer with a sheet resistance of $377 \Omega / s q$ is deposited. The bottom structure consists of a nearly perfect reflecting metal layer (Ruß et al., 2007). To increase the thermal resistance the membrane is fixed by two small legs (Fig. 2). The optical distance between membrane and reflection metal reaches an optimum for one quarter of the radiation wavelength. To reduce thermal losses by gas conduction a vacuum package is required. The bolometer converts the infrared radiation into heat energy and this induces a temperature rise resulting in a change of the electrical resistance. Typical bolometers have pixel pitch values of $35 \mu \mathrm{m}$ or $25 \mu \mathrm{m}$. Figure 2 shows a bolometer array with $4 \times 4$ sensor elements.

\section{Short-time integrator}

In Sect. 1, the ideal integrator model is analysed. In Sect. 2, the real integrator circuit is presented, analysed and compared to the ideal model.

\subsection{Ideal model}

Figure 3 shows the ideal short-time integrator model in the time-domain. By applying a bias voltage pulse $v_{\text {Bias }}(t)=$ $V_{\mathrm{Bias}}$ to the bolometer resistor $R_{\mathrm{Bolo}}\left(T_{0}\right)$ the bolometer current $i_{\mathrm{Bolo}}(t)$ is generated and integrated at the integration capacitor $C_{\text {int }}$. Only a small part of the bolometer current depends on the IR-radiation, so a bias current $i_{\text {Bias }}(t)=I_{\text {Bias }}$ is

Published by Copernicus Publications on behalf of the URSI Landesausschuss in der Bundesrepublik Deutschland e.V. 


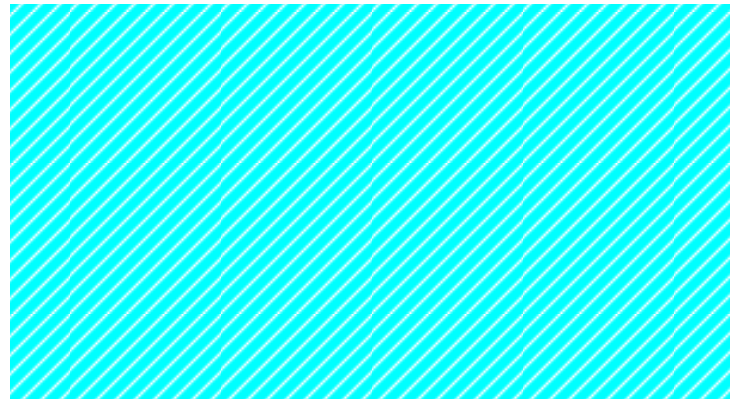

Fig. 1. Realization of a bolometer (cross section).

subtracted at the first summation node in order to cancel a corresponding offset at the integrator output. The integration stops after readout time $t_{\text {Pixel }}$. This is modelled in the following way: the second summation node subtracts the replica of the bolometer current delayed by the readout time $t_{\text {Pixel }}$ and integrates it. The integration constant is $-1 / C_{\text {int }}$. In this way we obtain a "time-windowed" integration. Otherwise the ideal integration would never end. The output voltage $V_{\text {out }}(s)$ is calculated in the Laplace-domain in Eq. (1).

$V_{\text {out }}(s)=\left(1-e^{-s t_{\text {Pixel }}}\right) \frac{-1}{s C_{\text {int }}}\left(\frac{U_{\text {Bias }}(s)}{R_{\text {Bolo }}\left(T_{0}\right)}-I_{\text {Bias }}(s)\right)$

Thus the transfer functions for output voltage $V_{\text {out }}(s)$ over input voltage $V_{\mathrm{Bias}}(s)$ and input current $I_{\mathrm{Bias}}(s)$ are given by Eqs. (2) and (3).

$$
\begin{aligned}
& H_{\text {in }, \text { ideal }}(s)=\frac{V_{\text {out }}(s)}{V_{\text {Bias }}(s)}=\left(1-e^{-s t_{\text {Pixel }}}\right) \frac{-1}{s R_{\text {Bolo }}\left(T_{0}\right) C_{\text {int }}} \\
& H_{\text {in2,ideal }}(s)=\frac{V_{\text {out }}(s)}{I_{\text {Bias }}(s)}=\left(1-e^{-s t_{\text {Pixel }}}\right) \frac{-1}{s C_{\text {int }}}
\end{aligned}
$$

\subsection{Real integrator}

Figure 4 shows the real readout integrated circuit (ROIC) consisting of an OTA with an integration capacitor $C_{\text {int }}$ in the feedback and all relevant noise sources. The output resistance is assumed to be infinity. The single-stage OTA is compensated by a load capacitance $C_{L}$. The integrator is reseted by the switch "reset". A parasitic capacitor $C_{p}$ caused e.g. by a column line is placed at the inverting input of the OTA. After the reset phase bolometer and a current source are connected to the inverting OTA input by the switch "select" for the readout time $t_{\text {Pixel }}$. The current from the current source is a constant during the integration, but there is no current if the switch "select" is off. Then the output voltage is held and sampled by a following stage not shown here. The switch resistance $R_{s}$ in series with the bolometer resistor $R_{\text {Bolo }}\left(T_{0}\right)$ can be neglected, because $R_{\text {Bolo }}\left(T_{0}\right) \gg$ $R_{s}$. If the transconductance of the OTA is sufficiently high, so that $g_{\text {mOTA }} R_{\text {Bolo }}\left(T_{0}\right) \gg 1$ and $C_{\text {int }} g_{\text {mOTA }} R_{\text {Bolo }}\left(T_{0}\right) \gg C_{L}$, the output voltage $V_{\text {out }}(s)$ is given by

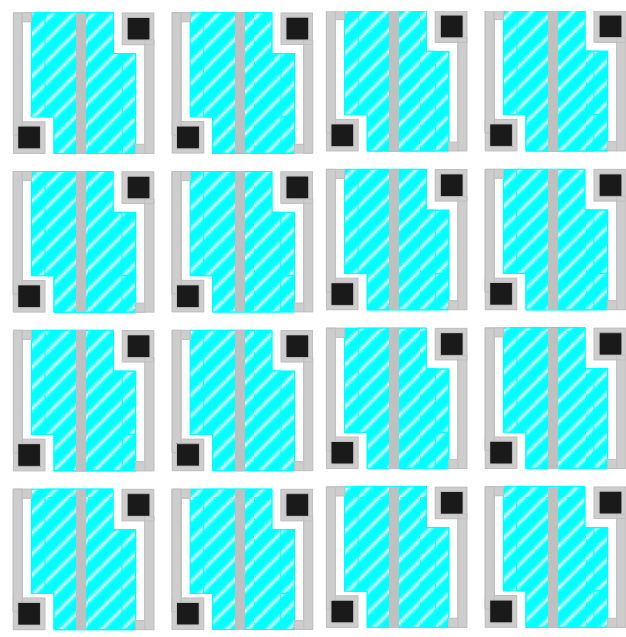

Fig. 2. Layout of a bolometer array.

$V_{\text {out }}(s)=\left(1-e^{-s t_{\mathrm{Pixel}}}\right) \cdot$
$\frac{V_{n, A}(s)\left(1+s\left(C_{\mathrm{int}}+C_{p}\right) R_{\mathrm{Bolo}}\left(T_{0}\right)\right)+\left(I_{\text {Bias }}(s) R_{\mathrm{Bolo}}\left(T_{0}\right)-V_{\text {Bias }}(s)\right)\left(1-\frac{s C_{\text {int }}}{g_{\text {mOTA }}}\right)}{s C_{\text {int }} R_{\text {Bolo }}\left(T_{0}\right)\left(1+\frac{s C_{e}}{g_{\text {mOTA }}}\right)}$

with

$C_{e}=C_{L}+C_{p}+\frac{C_{L} C_{p}}{C_{\text {int }}}$.

From Eq. (4) 3 transfer functions can be derived:

$$
\begin{aligned}
& H_{\text {in } 1}(s)=\frac{V_{\text {out }}(s)}{V_{\text {Bias }}(s)} \\
& =\frac{-\left(1-\frac{s C_{\text {int }}}{g_{\text {mOTA }}}\right)}{s C_{\text {int }} R_{\text {Bolo }}\left(T_{0}\right)\left(1+\frac{s C_{e}}{g_{\text {mOTA }}}\right)}\left(1-e^{-s t_{\text {Pixel }}}\right) \\
& H_{\text {in2 }}(s)=\frac{V_{\text {out }}(s)}{I_{\text {Bias }}(s)}=\frac{\left(1-\frac{s C_{\text {int }}}{g_{\text {mOTA }}}\right)}{s C_{\text {int }}\left(1+\frac{s C_{e}}{g_{\text {mOTA }}}\right)}\left(1-e^{\left.-s t_{\text {Pixel }}\right)}\right. \\
& H_{\text {in } 3}(s)=\frac{V_{\text {out }}(s)}{V_{n, A}(s)} \\
& =\left(\frac{1}{s C_{\text {int }} R_{\text {Bolo }}\left(T_{0}\right)}+1+\frac{C_{p}}{C_{\text {int }}}\right) \frac{1}{1+\frac{s C_{e}}{g_{\text {mOTA }}}}\left(1-e^{-s t_{\text {Pixel }}}\right)
\end{aligned}
$$

$H_{\text {in } 1}(s)$ and $H_{\text {in } 2}(s)$ compared to $H_{\text {in1,ideal }}(s)$ and $H_{\text {in2, deal }}(s)$, respectively, shows that there is now a zero in the transfer function and an additional non-dominant pole, which is given by the gain bandwith product (GBW) of the OTA. If the zero is not dominant, its effect can be 


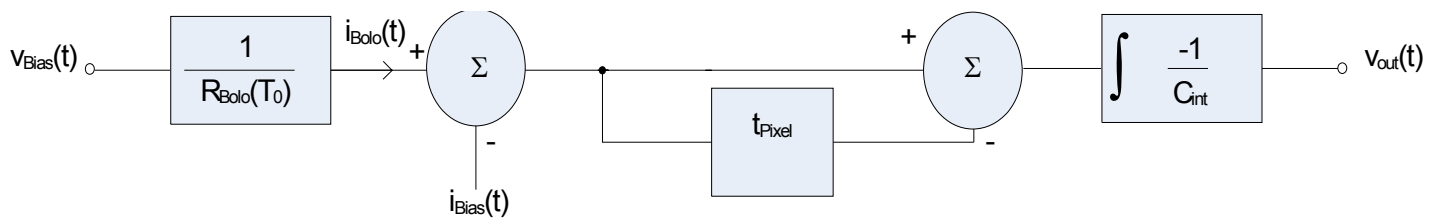

Fig. 3. Ideal integrator-model.

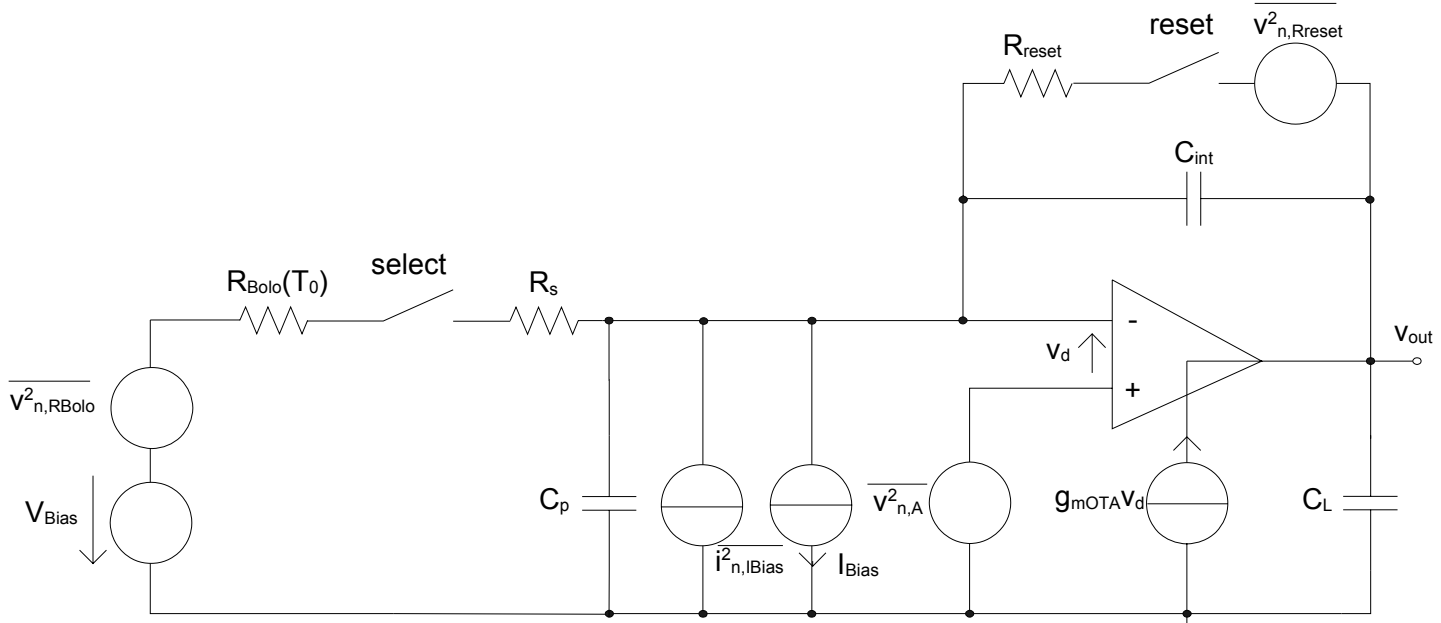

Fig. 4. Real ROIC with an OTA (noise sources included).

neglected. But $H_{\mathrm{in} 3}(s)$ is of special interest. Noise at the noninverting input of the OTA is integrated but additionally amplified by $\left(1+C_{p} / C_{\text {int }}\right)$ up to the GBW. There could be a high amplification if there is a large parasitic capacitance and a small integration capacitance with a large bandwith dependent on OTA's GBW.

\section{Noise calculation and NETD}

The power noise density $\mathrm{S}_{R \text { Bolo }}$ including white and $1 / f$ noise for the bolometer resistor is given by

$$
\begin{aligned}
& S_{\text {RBolo }}(f)=\frac{\overline{v_{n, \text { RBolo }, w}^{2}}}{\Delta f}+\frac{\overline{v_{n, \text { RBolo }, 1 / f}^{2}}}{\Delta f} \\
& =4 k T_{0} R_{\text {Bolo }}\left(T_{0}\right)+V_{\text {Bias }}^{2} \frac{k_{\text {fBolo }}}{f}
\end{aligned}
$$

with $k$ as Boltzman's constant, $k_{\mathrm{fBolo}}$ the $1 / f$-noise parameter and $T_{0}$ as the reference temperature. The power noise density $S_{A}$ including white and $1 / f$-noise for the OTA is given by

$$
\begin{aligned}
& S_{A}(f)=\overline{\frac{v_{n, A, w}^{2}}{\Delta f}}+\frac{\overline{v_{n, A, 1 / f}^{2}}}{\Delta f} \\
& =2 \frac{4 K_{a} k T_{0} n \gamma}{g_{\text {mOTA }}}+2 K_{b} \frac{k_{\text {fOTA }}}{W L C_{o x}^{2} f}
\end{aligned}
$$

with $W$ as the width of the input transistor, $L$ as the length of the input transistor, $C_{o x}$ as the capacitance per unit of the transistor, $n$ the weak inversion slope parameter, $\gamma$ as the noise parameter and $k_{\mathrm{fOTA}}$ as the $1 / f$-noise parameter of the input transistor (Anelli, 2000). $K_{a}$ and $K_{b}$ account for the effect of OTA's remaining transistors on total OTA's white noise and $1 / f$-noise respectively. The power noise density of the current source depends on the realization. In the simplest case it could be a resistor with the same resistance as the bolometer resistance. To make it easy it is assumed that the current source is ideal, but has a power noise density given by

$S_{\text {IBias }}=\frac{\overline{i_{n, \text { IBias }, w}^{2}}}{\Delta f}=\frac{4 k T_{0}}{R_{\text {Bolo }}\left(T_{0}\right)}$.

At first the noise power at the integrator output caused by the bolometer for white noise is calculated. 


$$
\begin{aligned}
& \overline{v_{n, \text { out }, \mathrm{RBolo}, w}^{2}}=\int_{f=0 H z}^{\infty} \frac{\overline{v_{n, \mathrm{Bolo}, w}^{2}}}{\Delta f}\left|H_{\mathrm{in} 1}(f)\right|^{2} d f=\int_{f=0 H z}^{\infty} 4 k T_{0} R_{\mathrm{Bolo}}\left(T_{0}\right)\left(\frac{2 \sin \left(\pi f t_{\text {Pixel }}\right)}{2 \pi f R_{\mathrm{Bolo}}\left(T_{0}\right) C_{\text {int }}}\right)^{2} \cdot \frac{1}{1+\left(2 \pi f \frac{C_{e}}{g_{\text {mOTA }}}\right)^{2}} d f+ \\
& \int_{f=0 H z}^{\infty} 4 k T_{0} R_{\text {Bolo }}\left(T_{0}\right)\left(\frac{2 \sin \left(\pi f t_{\text {Pixel }}\right)}{2 \pi f R_{\text {Bolo }}\left(T_{0}\right) C_{\text {int }}}\right)^{2} \cdot \frac{\left(2 \pi f \frac{C_{\text {int }}}{g_{\text {mOTA }}}\right)^{2}}{1+\left(2 \pi f \frac{C_{e}}{g_{\text {mOTA }}}\right)^{2}} d f \\
& \approx \int_{f=0 H z}^{\infty} 4 k T_{0} R_{\text {Bolo }}\left(T_{0}\right)\left(\frac{2 \sin \left(\pi f t_{\text {Pixel }}\right)}{2 \pi f R_{\text {Bolo }}\left(T_{0}\right) C_{\text {int }}}\right)^{2} d f+\int_{f=0 H z}^{\infty} 4 k T_{0} R_{\text {Bolo }}\left(T_{0}\right)\left(\frac{2}{2 \pi f R_{\text {Bolo }}\left(T_{0}\right) C_{\text {int }}}\right)^{2} \cdot \frac{\left(2 \pi f \frac{C_{\text {int }}}{g_{\text {mOTA }}}\right)^{2}}{1+\left(2 \pi f \frac{C_{e}}{g_{\text {mOTA }}}\right)^{2}} d f \\
& \approx 2 k T_{0}\left(\frac{t_{\text {Pixel }}}{R_{\text {Bolo }}\left(T_{0}\right) C_{\text {int }}^{2}}+\frac{2}{g_{\text {mOTA }} R_{\text {Bolo }}\left(T_{0}\right) C_{e}}\right)
\end{aligned}
$$

This result has been obtained using several approximations. The noise power at the output caused by the current source is calculated similarly as:

$\overline{v_{n, \text { out }, \text { IBias }, w}^{2}}=\int_{f=0 H z}^{\infty} \frac{\overline{i_{n, \text { IBias }, w}^{2}}}{\Delta f}\left|H_{\text {in2 } 2}(f)\right|^{2} d f \approx 2 k T_{0}\left(\frac{t_{\text {Pixel }}}{R_{\text {Bolo }\left(T_{0}\right) C_{\text {int }}^{2}}}+\frac{2}{g_{\text {mOTA }} R_{\text {Bolo }}\left(T_{0}\right) C_{e}}\right)$.

The noise power caused by OTA's white noise is determined as

$$
\begin{aligned}
& \overline{v_{n, \text { out }, A, w}^{2}}=\int_{f=0 H z}^{\infty} \frac{\overline{v_{n, A, w}^{2}}}{\Delta f}\left|H_{\mathrm{in} 3}(f)\right|^{2} d f \approx \int_{f=0 H z}^{\infty} 2 \frac{4 K_{a} k T_{0} n \gamma}{g_{\mathrm{mOTA}}}\left[\left(\frac{1}{R_{\mathrm{Bolo}}\left(T_{0}\right) C_{\mathrm{int}}} \cdot \frac{2 \sin \left(\pi f t_{\text {Pixel }}\right)}{2 \pi f}\right)^{2}+2 \frac{\left(1+\frac{C_{P}}{C_{\text {int }}}\right)^{2}}{1+\left(2 \pi f \frac{C_{e}}{g_{\mathrm{mOTA}}}\right)^{2}}\right] d f \\
& \approx \frac{4 K_{a} k T_{0} n \gamma}{g_{\mathrm{mOTA}}} \frac{t_{\mathrm{Pixel}}}{\left(R_{\mathrm{Bolo}}\left(T_{0}\right) C_{\mathrm{int}}\right)^{2}}+2\left(1+\frac{C_{P}}{C_{\mathrm{int}}}\right)^{2} \frac{2 K_{a} k T_{0} n \gamma}{C_{e}} .
\end{aligned}
$$

The noise power caused by the bolometer resistor's $1 / f$-noise is described as

$$
\begin{aligned}
& \overline{v_{n, \text { out }, \text { RBolo }, 1 / f}^{2}}=\int_{f_{1}}^{\infty} \overline{\frac{v_{n, \text { Bolo }, 1 / f}^{2}}{\Delta f}}\left|H_{\text {in } 1}(f)\right|^{2} d f=\int_{f_{1}}^{\infty} V_{\text {Bias }}^{2} \frac{k_{\text {fBolo }}}{f}\left(\frac{1}{R_{\text {Bolo }}\left(T_{0}\right) C_{\text {int }}} \cdot \frac{2 \sin \left(\pi f t_{\text {Pixel }}\right)}{2 \pi f}\right)^{2} \cdot \frac{1}{1+\left(2 \pi f \frac{C_{e}}{g_{\text {mOTA }}}\right)^{2}} d f+ \\
& \int_{f_{1}}^{\infty} V_{\text {Bias }}^{2} \frac{k_{\text {fBolo }}}{f}\left(\frac{1}{R_{\text {Bolo }}\left(T_{0}\right) C_{\text {int }}} \cdot \frac{2 \sin \left(\pi f t_{\text {Pixel }}\right)}{2 \pi f}\right)^{2} \cdot \frac{\left(2 \pi f \frac{C_{\text {int }}}{g_{\text {mOTA }}}\right)^{2}}{1+\left(2 \pi f \frac{C_{e}}{g_{\text {mOTA }}}\right)^{2}} d f \\
& \approx V_{\text {Bias }}^{2} k_{\mathrm{fBolo}}(-1) \frac{-1+\cos (\xi)-\xi \sin (\xi)+\xi^{2} C i(\xi)}{\xi^{2} R_{\text {Bolo }}^{2}\left(T_{0}\right) C_{\text {int }}^{2}} t_{\text {pixel }}^{2}+\frac{V_{\text {Bias }}^{2} k_{\text {fBolo }}}{\left(g_{\text {mOTA }} R_{\text {Bolo }}\left(T_{0}\right)\right)^{2}} \ln \left(\frac{\sqrt{1+\left(2 \pi f_{1} \frac{C_{e}}{g_{\text {mOTA }}}\right)^{2}}}{\left(2 \pi f_{1} \frac{C_{e}}{g_{\text {mOTA }}}\right)}\right) \\
& \approx V_{\text {Bias }}^{2} k_{\mathrm{fBolo}}\left(\frac{t_{\text {Pixel }}}{R_{\text {Bolo }}\left(T_{0}\right) C_{\text {int }}}\right)^{2} \ln \left(\frac{1}{2 \pi f_{1} t_{\text {Pixel }}}\right)+\frac{1}{\left(g_{\text {mOTA }} R_{\text {Bolo }}\left(T_{0}\right)\right)^{2}} \ln \left(\frac{1}{\left(2 \pi f_{1} \frac{C_{e}}{g_{\text {mOTA }}}\right)}\right) .
\end{aligned}
$$

where $\xi=2 \pi t_{\text {Pixel }} f_{1}, \quad \cos (\xi) \approx 1, \quad \sin (\xi) \approx \xi, \quad$ the Cosine Integral $\operatorname{Ci}(\xi) \approx \ln (\xi)$ with $|\ln (\xi)| \gg 1$ and $2 \pi f_{1} C_{e} / g_{\text {mOTA }} \ll 1$. A mechanical shutter in front of the IRFPA is periodically closed. This can be modelled as a high pass filter. The lower frequency $f_{1}$ is assumed to be (Wood, 1997)

$$
f_{1} \approx \frac{1}{4 t_{\text {shutter }}} .
$$


$t_{\text {shutter }}$ is the shutter time, where $t_{\text {shutter }} \gg t_{\text {Pixel }}$. The contribution of the $1 / f$-noise of the OTA with same approximations as above is given by

$$
\begin{aligned}
& \overline{v_{n, \text { out }, A, 1 / f}^{2}}=\int_{f_{1}}^{\infty} \frac{\overline{v_{n, A, 1 / f}^{2}}}{\Delta f}\left|H_{\mathrm{in} 3}(f)\right|^{2} d f \\
& \approx \int_{f_{1}}^{\infty} 2 K_{b} \frac{k_{\text {fOTA }}}{W L C_{\text {ox }}^{2} f}\left(\begin{array}{l}
\frac{\left(2 \sin \left(\pi f t_{\text {Pixel }}\right)\right)^{2}}{\left(2 \pi f R_{\text {Bolo }}\left(T_{0}\right) C_{\text {int }}\right)^{2}}+ \\
+\left(1+\frac{C_{P}}{C_{\text {int }}}\right)^{2} \frac{4}{1+\left(2 \pi f \frac{C_{e}}{g_{\text {mOTA }}}\right)^{2}}
\end{array}\right) d f \\
& \approx 2 K_{b} \frac{k_{\text {fOTA }}}{W L C_{\text {ox }}^{2}}\left(\begin{array}{l}
\left(\frac{t_{\text {Pixel }}}{R_{\text {Bolo }}\left(T_{0}\right) C_{\text {int }}}\right)^{2} \ln \left(\frac{1}{2 \pi t_{\text {Pixel }} f_{1}}\right)+ \\
4\left(1+\frac{C_{P}}{C_{\text {int }}}\right)^{2} \ln \left(\frac{1}{\left(2 \pi f_{1} \frac{C_{e}}{g_{\text {mOTA }}}\right)}\right)
\end{array}\right) .
\end{aligned}
$$

During the reset of the integrator the kTC-noise is stored on the integration capacitor. Thus

$\overline{v_{n, \text { reset }}^{2}}=\frac{k T_{0}}{C_{\text {int }}}$.

The current responsitivity of the bolometer at the input is given for a constant voltage across the bolometer by

$R_{I} \approx \frac{-V_{\text {Bias }} \alpha}{R_{\text {Bolo }}\left(T_{0}\right) g_{\text {Bolo }}}$.

where $\alpha$ is the temperature coefficient (TCR) and $g_{\text {Bolo is }}$ the heat conductance of the bolometer (derived from Wood, 1997 ) with $g_{\text {Bolo }} \approx g_{\text {eff }}, I_{\text {Bolo }}=V_{\text {Bias }} / R_{\text {Bolo }}\left(T_{0}\right), \alpha<0, I_{\text {Bolo }}$ as the constant bolometer current, $g_{\text {eff }}$ as the effective heat conductance). The TCR $\alpha$ is assumed to be constant. The total output noise power is refered to the input by multiplying by the square of the DC value of the transferfunction $H_{\text {in } 1}(f)$ and converted into a current by division by $R_{\text {Bolo }}^{2}\left(T_{0}\right)$. The NETD is defined as

$\mathrm{NETD}=\frac{4 \cdot F_{n o}^{2}}{\pi \cdot A_{\text {Bolo }} \cdot \varepsilon_{\text {Bolo }} \cdot \frac{d L}{d T_{\text {target }}}} \frac{\sqrt{\sqrt{i_{n, \text { total }}^{2}}}}{R_{I}}$

where $i_{n \text {,total }}$ is the total noise current, $F_{n o}$ is the f-number of the optic, $A_{\text {Bolo }}$ the area of the bolometer, $\varepsilon_{\text {Bolo }}$ is the emission coefficient which is equal to the absorption coefficient and $d L / d T_{\text {target }}$ is the differential change of radiance over the change of the target temperature (Wood, 1997). Finally, total NETD is calculated to

$$
\begin{aligned}
& \mathrm{NETD}=\frac{4 \cdot F_{n o}^{2}}{\pi \cdot A_{\mathrm{Bolo}} \cdot \varepsilon_{\mathrm{Bolo}} \cdot \frac{d L}{d T_{t} \arg e t}} \cdot\left(\frac{1}{R_{\mathrm{Bolo}\left(T_{0}\right)}}\right) \cdot\left(\frac{R_{\mathrm{Bolo}\left(T_{0}\right) C_{\mathrm{int}}}}{t_{\mathrm{Pixel}}}\right) .
\end{aligned}
$$

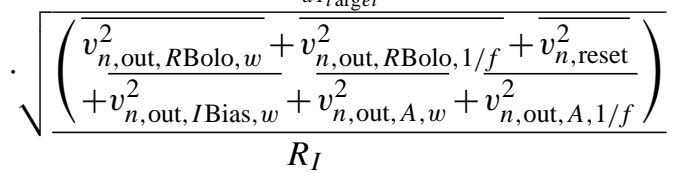

Assuming that there is only noise from the bolometer resistor (white and $1 / f$-noise) and current source (white noise) with $g_{\text {mOTA }} \rightarrow \infty, C_{L} \rightarrow \infty, \mathrm{WL} \rightarrow \infty$ the NETD reaches

$\mathrm{NETD}=\frac{4 \cdot F_{\text {no }}^{2} g_{\text {Bolo }}}{\pi \cdot A_{\text {Bolo }} \cdot \varepsilon_{\text {Bolo }} \cdot \frac{d L}{d T_{\text {t }} \text { arget }}} \cdot \frac{\sqrt{\frac{4 k T_{0} R_{\text {Bolo }}\left(T_{0}\right)}{{ }^{t_{\text {Pixel }}}+V_{\text {Bias }}^{2} k_{\text {fBolo }} \ln \left(\frac{2 t_{\text {shutter }}}{\pi t_{\text {Pixel }}}\right)}}}{\left|-V_{\text {Bias }} \alpha\right|}$.

NETD caused by white noise can be decreased by increasing the readout time and bias voltage and by decreasing the bolometer resistance assuming that the bolometer parameters and $\mathrm{f}$-number are fixed. NETD caused by $1 / f$-noise is independent of the bias voltage, because it cancels out. Because of the logarithm there won't be much change when varying $t_{\text {Pixel }}$ for a fixed shutter time. Thus white noise can be reduced, but $1 / f$-noise cannot. So $1 / f$-noise will limit NETD.

\section{Conclusions}

The ideal and real short-time integrator have been modelled. The effect of the finite integration time has been included. The total noise power and the resulting NETD have been calculated. For long integration the limiting factor as far as NETD is concerned is $1 / f$-noise.

\section{References}

Ruß, M., Bauer, J., and Vogt, H.: The geometric design of microbolometer elements for uncooled focal plane arrays, Proc. of SPIE Conference Infrared Technology and Applications XXXIII, Volume 6542, 654223-1-10, 2007.

Anelli, G.: Design and Characterization of Radiation Tolerant Integrated Circuits in Deep Submicron CMOS Technologies for the LHC Experiments, Ph.D. Thesis, Institut National Polytechnique de Grenoble, France, 2000.

Wood, R. A.: Monolithic Silicon Microbolometer Arrays, in Uncooled Infrared Imaging Arrays and Systems, edited by: Kruse, P. and Skatrud, D., Semiconductors and Semimetals, 47, 45-121, Academic Press, 1997. 